\title{
Inside Plastic Stents versus Metal Stents for Treating Unresectable Malignant Perihilar Biliary Obstructions: A Retrospective Comparative Study
}

\author{
Yoshihide Kanno', Shinsuke Koshita', Takahisa Ogawa', Hiroaki Kusunose', Kaori Masu', Toshitaka Sakai', Keisuke Yonamine', \\ Kazuaki Miyamoto', Toji Murabayashi ${ }^{1}$, Fumisato Kozakai ${ }^{1}$, Jun Horaguchi ${ }^{1,2}$, Yutaka Noda ${ }^{1}$ and Kei Ito ${ }^{1}$ \\ ${ }^{1}$ Department of Gastroenterology, Sendai City Medical Center, Sendai, ${ }^{2}$ Natori Chuo Clinic, Sendai, Japan
}

Background/Aims: The aim of this study was to evaluate outcomes of inside plastic stents (iPSs) versus those of metal stents (MSs) for treating unresectable perihilar malignant obstructions.

Methods: For all patients who underwent endoscopic suprapapillary placement of iPS(s) or MS(s) as the first permanent biliary drainage for unresectable malignant perihilar obstructions between January 2014 and August 2019, clinical outcomes using iPSs ( $n=20$ ) and MSs $(n=85)$, including clinical efficacy, adverse events, and time to recurrence of biliary obstruction (RBO), were retrospectively evaluated.

Results: There were no differences in clinical effectiveness (95\% for the iPS group vs. $92 \%$ for the MS group, $p=1.00$ ). Procedure-related adverse events, including pancreatitis, acute cholangitis, acute cholecystitis, and death, were observed for $8 \%$ of the MS group, although no patient in the iPS group developed such adverse events. The median time to RBO was 561 days ( $95 \%$ confidence interval, $0-1,186$ days) for iPSs and 209 days (127-291 days) for MSs, showing a significant difference ( $p=0.008)$.

Conclusions: Time to RBO after iPS placement was significantly longer than that after MS placement. IPSs, which are removable, unlike MSs, were an acceptable option. Clin Endosc 2020;53:735-742

Key Words: Above the papilla; Benign stricture; Bile duct cancer; Endoscopic retrograde cholangiopancreatography; Hilar cholangiocarcinoma

\section{INTRODUCTION}

Even in the field of pancreatobiliary endoscopy, which has been rapidly developing, drainage methodology for use with perihilar biliary obstructions remains one of the most difficult issues to overcome. Perihilar obstruction separates the biliary tree into numerous cavities that cannot be drained using cur-

Received: November 29, 2019 Revised: December 27, 2019

Accepted: January 4, 2020

Correspondence: Yoshihide Kanno

Department of Gastroenterology, Sendai City Medical Center, Tsurugaya 5-22-1, Miyagino-ku, Sendai 983-0824, Japan

Tel: +81-22-252-1111, Fax: +81-22-252-9431, E-mail: yoshi-hk@openhp.or.jp ORCID: https://orcid.org/0000-0002-2044-5483

cc This is an Open Access article distributed under the terms of the Creative Commons Attribution Non-Commercial License (http://creativecommons.org/ licenses/by-nc/3.0) which permits unrestricted non-commercial use, distribution, and reproduction in any medium, provided the original work is properly cited. rent drainage techniques when they are too small or numerous. Incomplete drainage, segmental infection, biloma, and/ or partial but prolonged liver damage often occur, resulting in quality of life (QOL) deterioration and decreased survival. Even if such obvious events do not develop, drainage stents can become occluded or insufficient in a relatively short interval.

Since the advent of self-expanding metal stents (MSs), clinical outcomes of metal versus plastic stents (PSs) have been compared. Although short-term efficacies, such as technical feasibility and drainage efficacy seem similar, the stent patency period was reportedly better for MSs in several studies, ${ }^{1-3}$ including a meta-analysis. ${ }^{4}$ However, for perihilar obstructions, all previous studies compared suprapapillarily placed MSs with transpapillarily placed PSs. Transpapillary placement, in which the distal end of the stent is within the duodenum, is 
associated with the free reflux of intestinal juices, food, and bacteria.

Inside plastic stents (iPSs), which are placed suprapapillarily, can theoretically prevent such reflux, and they can be compared with MSs placed above the papilla. If iPSs have an acceptable efficacy and patency period, they would be a potent option, particularly since MSs are unremovable.

Although there are several reports on iPS use for treating benign strictures ${ }^{5,6}$ and preoperative cancers, ${ }^{7}$ few studies have reported on their use for unresectable malignant perihilar strictures, except for single-arm evaluations of $\mathrm{iPSs}^{8,9}$ and studies comparing iPSs with transpapillary PSs. ${ }^{10}$ No study has compared the use of iPSs and MSs for treating perihilar malignancies.

Although prospective randomized studies are ideal for evaluating outcomes, here we conducted a retrospective comparative study as a pilot study to confirm the feasibility and acceptability of iPS placement.

\section{MATERIAL AND METHODS}

\section{Patients}

This retrospective study aimed to evaluate the clinical outcomes of iPSs and MSs for biliary stenting. All patients who underwent biliary drainage for unresectable perihilar malignancy using iPSs or MSs between January 2014 and August 2019 were extracted from a prospectively maintained endoscopic retrograde cholangiopancreatography (ERCP) database. To evaluate the outcomes of stent placement, cases of stent exchange or addition after the dysfunction of previously deployed stents were excluded, although cases of planned exchange of previously deployed stents as a temporary stopgap remedy until confirmation of inoperability or recovery from critical cholangitis were not excluded. Thus, even the first MS placement for cases in which previous iPSs became dysfunctional were not included into the analyses and vice versa. In addition, the following cases were excluded: (1) stricture classified as Bismuth level I, (2) biliary drainage with mixed usage of an iPS and an MS, (3) biliary drainage using a hybrid of multiple routes, such as transpapillary + percutaneous routes and transpapillary + endoscopic ultrasonography-guided routes, and (4) surgically reconstructed biliary systems, e.g., after surgical choledochojejunostomy.

\section{Endoscopic procedures}

After a contrast cholangiogram was obtained using a catheter cannulated through a duodenoscope (TJF260V; Olympus Co., Tokyo, Japan), guidewires were inserted into all cavities to be drained. Drainage cavities were defined using computed tomography and cholangiography according to drainage efficiency with the liver volume of the basin involved by the cavities. Stents used were determined at the endoscopist's discretion.

For iPS placement, a 3-0 nylon thread was tied to the end of the PS (Flexima; Boston Scientific Japan K.K., Tokyo, Japan,
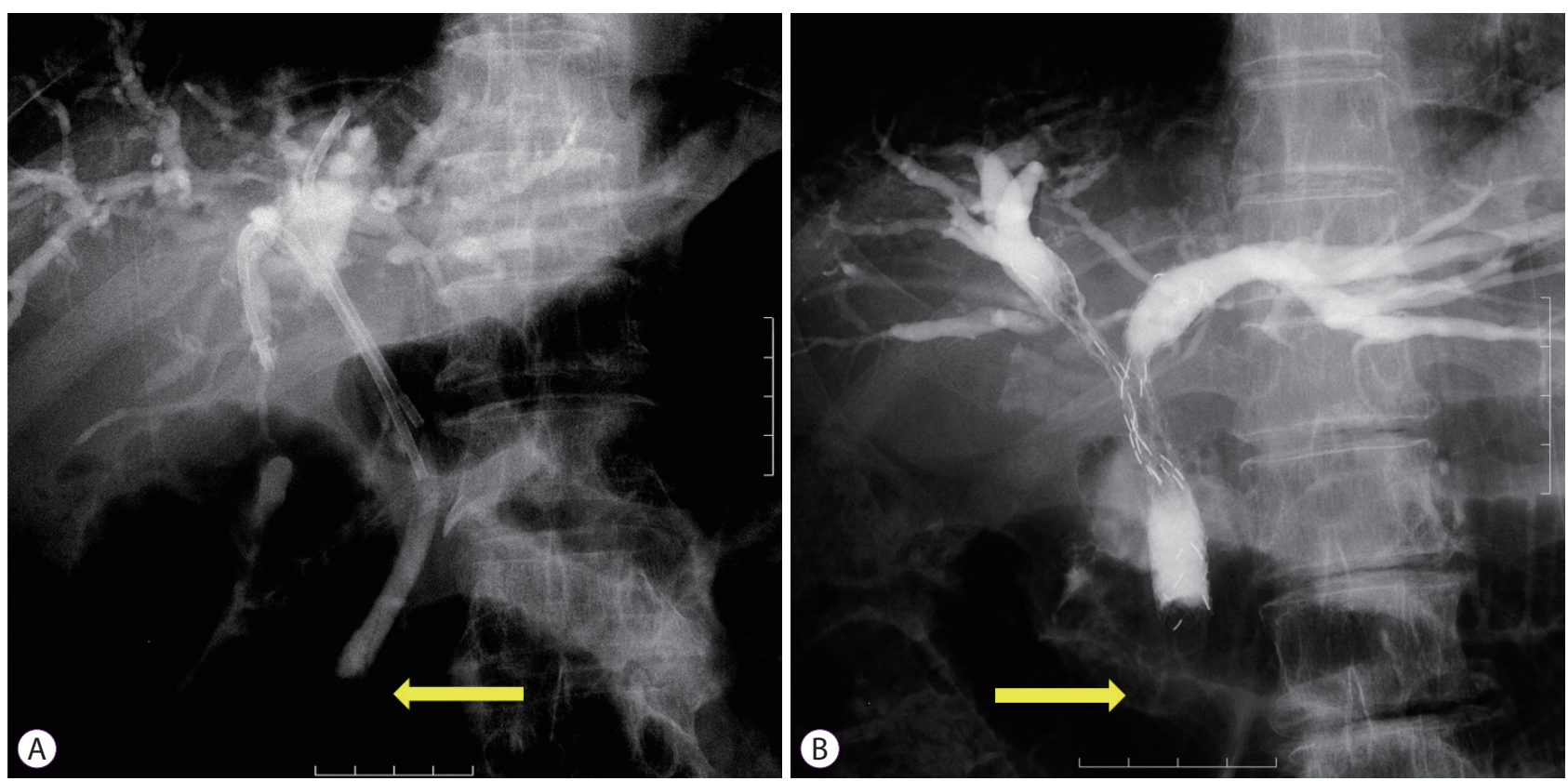

Fig. 1. Inside stents placed above the papilla (arrows). (A) Inside plastic stents; and (B) inside metal stents placed using a partial stent-in-stent method. 
Through Pass; Gadelius Medical, Tokyo, Japan, Quick Place V; Olympus) to aid in removal. The stent was disconnected from the delivery system to tie the thread and then reloaded into the same delivery system for insertion. Stents with a preloaded thread (Through \& Pass IS; Gadelius Medical) were used after becoming available. The iPS length was sufficiently long to avoid migration into the upstream cavity but short enough to avoid dislocation toward the duodenum beyond the papilla (Fig. 1A).

Multiple MSs were placed using a partial stent-in-stent (pSIS) or side-by-side (SBS) method at the endoscopist's discretion. When three or more stents were required, the pSIS method was applied (three or more stents were not placed using the SBS method or using a hybrid of the two methods). During the pSIS method, stents were placed in decreasing order of expected placement difficulty based on the angle between the common bile duct and the target duct, accessibility of a guidewire to the target, and stenosis length and severity (Fig. 1B). When it was difficult to place the stent(s) through the previously deployed stent, multiple endoscopy sessions were permitted. MSs used in the pSIS method were Niti-S LCD type (Century Medical, Inc., Tokyo, Japan), BonaStent M-Hilar (Medico’s Hirata Inc., Tokyo, Japan), Zilver 635 (Cook Japan Inc., Tokyo, Japan), BileRush (Piolax Medical Devices, Inc., Yokohama, Japan), and ZeoStent V (Zeon Medical Inc., Tokyo, Japan). For SBS placement, stents were simultaneously deployed using stents with a thin delivery system of 6 Fr or less (Zilver 635, BileRush, or ZeoStent V). The proximal (duodenal side) ends of the stents were positioned at the same level.

Endoscopic sphincterotomy (EST) was performed when the papilla was small, the biliary cannulation was difficult, the risk for post-procedural pancreatitis was considered high, or another intervention requiring EST, such as stone removal and cholangioscopy, was required.

Before stent placement, the obstructed cavities were aspirated and injected with saline a few times.

\section{Outcome measurements and definitions}

The primary measurement was the time to recurrence of biliary obstruction (RBO) defined as (1) elevation of the serum total bilirubin (TB) level to $>2.0 \mathrm{mg} / \mathrm{dL}$ from a normal level ( $\leq 1.2 \mathrm{mg} / \mathrm{dL}$ ), (2) elevation of the serum TB level of $>2.0$ $\mathrm{mg} / \mathrm{dL}$ from an abnormal level (>1.3 mg/dL), or (3) the occurrence of acute infectious cholangitis with an elevated C-reactive protein (CRP) level, an elevated biliary enzyme level, and pyrexia. RBO was evaluated only for patients in whom iPS or MS placement was clinically effective. Clinical effectiveness was defined as successful recovery from obstructive jaundice and infectious cholangitis. Successful recovery from obstruc- tive jaundice was defined as (1) serum TB level decreasing to normal; or (2) serum TB level decreasing to $<1 / 3$ of the peak level. Successful recovery from infectious cholangitis was defined as no increase in the serum CRP and biliary enzyme levels without antibiotic agent administration for $>7$ days.

Clinical effectiveness, procedure-related adverse events, late biliary complications except RBO, and survival period were analyzed as secondary outcomes. Procedure-related adverse events were defined as all clinically unfavorable events occurring within 7 days, while late biliary complications were clinically unfavorable events in the liver/biliary system $>7$ days after the procedure. When multiple sessions were needed to complete the drainage, dates were counted from the last session.

Table 1. Baseline Characteristics

\begin{tabular}{|c|c|c|c|}
\hline & $\begin{array}{l}\text { iPS group } \\
(n=20)\end{array}$ & $\begin{array}{l}\text { MS group } \\
(n=85)\end{array}$ & $p$-value \\
\hline Sex, Male:Female & $8: 12$ & $51: 34$ & 0.13 \\
\hline Age, mean $\pm S D, y r$ & $79 \pm 8$ & $79 \pm 11$ & 0.85 \\
\hline Etiology & & & n.s. \\
\hline $\begin{array}{l}\text { Extrahepatic cholangio- } \\
\text { carcinoma }\end{array}$ & $9(45 \%)$ & $42(49 \%)$ & \\
\hline $\begin{array}{l}\text { Intrahepatic cholangio- } \\
\text { carcinoma }\end{array}$ & $3(15 \%)$ & $16(19 \%)$ & \\
\hline Gallbladder cancer & $3(15 \%)$ & $12(14 \%)$ & \\
\hline Other & $5(25 \%)$ & $15(18 \%)$ & \\
\hline Bismuth level & & & n.s. \\
\hline II & $7(35 \%)$ & $17(20 \%)$ & \\
\hline IIIa, IIIb & $6(30 \%)$ & $27(31 \%)$ & \\
\hline IV & $7(35 \%)$ & $41(48 \%)$ & \\
\hline $\begin{array}{l}\text { Rough image of the stric- } \\
\text { ture }^{11}\end{array}$ & & & 0.50 \\
\hline Localized & $16(80 \%)$ & $73(86 \%)$ & \\
\hline Spreading & $4(20 \%)$ & $12(14 \%)$ & \\
\hline $\begin{array}{l}\text { Surface image of the stric- } \\
\text { ture }^{11}\end{array}$ & & & n.s. \\
\hline Soft irregularity & $12(60 \%)$ & $32(38 \%)$ & \\
\hline Solid irregularity & $7(35 \%)$ & $46(54 \%)$ & \\
\hline Smooth & $1(5 \%)$ & $7(8 \%)$ & \\
\hline Infectious cholangitis & & & n.s. \\
\hline None & $9(45 \%)$ & $25(29 \%)$ & \\
\hline None but previous & $1(5 \%)$ & $13(15 \%)$ & \\
\hline Concomitant & $10(50 \%)$ & $47(55 \%)$ & \\
\hline
\end{tabular}

iPS, inside plastic stent; MS, metal stent; n.s., not significant; SD, standard deviation. 


\section{Analytic statistics}

Analyses were performed using IBM SPSS software version 24 (IBM Japan, Ltd., Tokyo, Japan). Outcomes are reported as mean \pm standard deviation for quantitative variables and as percentages for categorical variables. Continuous variables were analyzed using a $t$-test, while categorical data were compared using Fisher's exact test. More than two categorical variables were analyzed using the chi-square test. Cumulative survival was analyzed using the Kaplan-Meier method. Median periods calculated using the Kaplan-Meier method are reported with $95 \%$ confidence intervals (CIs). $P$-values $<0.05$ were considered statistically significant.

\section{Ethics}

The stent placement procedures and this study were approved by the Institutional Review Board of Sendai City Medical Center. Written informed consent for ERCP was obtained from all patients before the procedure after an explanation of the risks and benefits versus other possible options was provided.

All authors had access to the study data and reviewed and approved the final manuscript.

\section{RESULTS}

\section{Baseline characteristics}

During the study period, 20 patients in the iPS group and 85 patients in the MS group were identified as being fully eligible for this study. The distributions of sex, age, and causative etiology were similar between the groups (Table 1). The rate of cases of a Bismuth level IV stricture was relatively high in the MS group, although no statistically significant differences

Table 2. Procedural Details

\begin{tabular}{lccc}
\hline & $\begin{array}{c}\text { iPS group } \\
(\boldsymbol{n}=\mathbf{2 0})\end{array}$ & $\begin{array}{c}\text { MS group } \\
(\boldsymbol{n}=\mathbf{8 5})\end{array}$ & $\boldsymbol{p}$-value \\
\hline EST & $6(30 \%)$ & $45(53 \%)$ & 0.08 \\
Number of stents & & & n.s. \\
1 & $7(35 \%)$ & $24(28 \%)$ & \\
2 & $10(50 \%)$ & $51(60 \%)$ & \\
3 & $2(10 \%)$ & $9(11 \%)$ & \\
4 & $1(5 \%)$ & $1(1 \%)$ & \\
Placing method & & & \\
Partial stent-in-stent & & $52(61 \%)$ & \\
Side by side & & $9(11 \%)$ & \\
\hline
\end{tabular}

EST, endoscopic sphincterotomy; iPS, inside plastic stent; MS, metal stent; n.s., not significant. detected. Rough and surface images on cholangiography were classified according to the criteria defined in our previous report. ${ }^{11}$ Although soft irregularities on the surface of the stric-

Table 3. Clinical Outcomes

\begin{tabular}{lccc}
\hline & $\begin{array}{c}\text { iPS group } \\
(\boldsymbol{n}=\mathbf{2 0})\end{array}$ & $\begin{array}{c}\text { MS group } \\
(\boldsymbol{n}=\mathbf{8 5})\end{array}$ & $\boldsymbol{p}$-value \\
\hline Clinical efficacy & $95 \%(19 / 20)$ & $92 \%(77 / 85)$ & 1.00 \\
Procedure-related adverse events & & \\
Overall & 0 & $8 \%(7 / 85)$ & 0.34 \\
Mild pancreatitis & & $(3)$ & \\
Acute cholangitis & & $(2)$ & \\
Acute cholecystitis & & $(1)$ & \\
Mortality & & $(1)$ &
\end{tabular}

Chemotherapy after the procedure

(with overlapping)

$\begin{array}{lcc}\begin{array}{l}\text { Performed } \\ \text { (any regimen) }\end{array} & 10(50 \%) & 28(33 \%) \\ \text { Gemcitabine } & 1 & 6 \\ \text { S-1 } & 4 & 10 \\ \text { Gemcitabine + } & 6 & 14 \\ \text { CDDP } & & 3 \\ \text { Other(s) } & 1 & \end{array}$

$\begin{array}{lccc}\text { Late complications } & & & \\ \text { Overall } & 10 \%(2 / 20) & 12 \%(10 / 85) & 1.00 \\ \text { Liver abscess } & (1) & (5) & \\ \begin{array}{c}\text { Acute cholecystitis } \\ \text { Stent dysfunction }\end{array} & 21 \%(4 / 19) & 43 \%(33 / 77) & 0.11 \\ \begin{array}{c}\text { Time to RBO, median } \\ \text { (95\% CI), days }\end{array} & 561(0-1,186) & 209(127-291) & 0.008\end{array}$

Number of re-interventions

n.s.

$\begin{array}{lccc}0 & 16 & 57 & \\ 1 & 3 & 14 & \\ 2 & 1 & 4 & \\ 3 & 0 & 1 & \\ 4 & 0 & 4 & \\ 5 & 0 & 3 & 0.31 \\ 8-9 & 0 & 2 & 0.68 \\ \text { Mean } \pm \text { SD } & 1.1 \pm 1.9 & 0.7 \pm 0.9 & \\ \text { Follow up period, } & 203 \pm 224 & 182 \pm 195 & 0.37 \\ \text { mean } \pm \text { SD, days } & 329(181-477) & 224(149-298) & 0 \\ \text { Survival period, me- } \\ \text { dian (95\% CI), days }\end{array}$

CDDP, cisplatin; CI, confidence interval; iPS, inside plastic stent; MS, metal stent; n.s., not significant; RBO, recurrent biliary obstruction; SD, standard deviation. 
ture were significantly related to short patency in the report, the intergroup distributions were not statistically different. Acute infectious cholangitis was observed in $50 \%$ of the iPS group and 55\% of the MS group. No significant tendencies for stent selection were observed between periods, which were divided into two and four phases, to determine if such tendencies were present.

\section{Short-term outcomes}

EST was performed in more patients in the MS group than in the iPS group without statistical significance (30\% vs. $53 \%$,

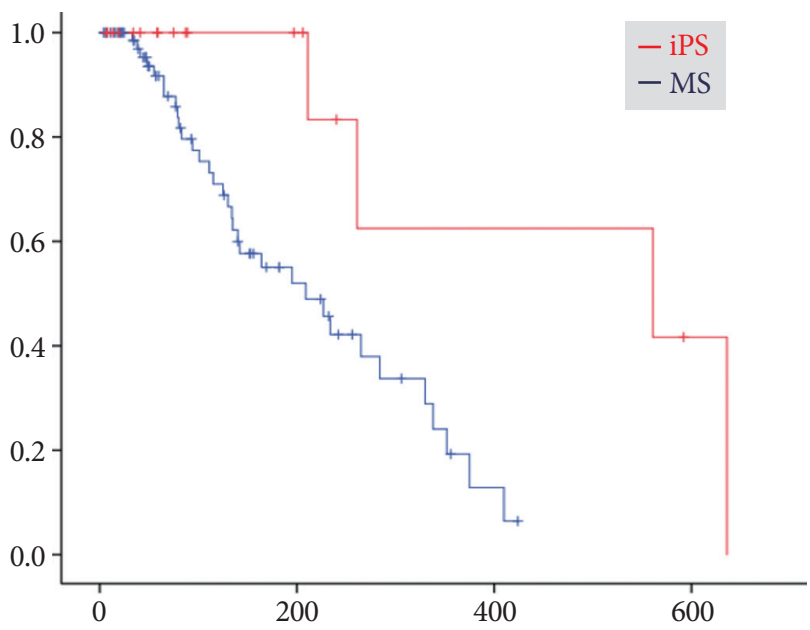

Fig. 2. Kaplan-Meier curves of cumulative rates to recurrent biliary obstruction (RBO). Median times to RBO were 561 (95\% confidence interval $[\mathrm{Cl}], 0-1,186)$ days for the inside plastic stent (iPS) group and $209(95 \% \mathrm{Cl}$, $127-291)$ days for the metal stent (MS) group $(p=0.008)$. $p=0.08$ ) (Table 2). Drainage required two or more stents for $65 \%$ of the iPS group and $72 \%$ of the MS group ( $p=0.59$ ). For most of the MS group (85\%, 52/61), MSs were placed using the pSIS method. The drainage was clinically effective for $95 \%$ $(19 / 20)$ of the iPS group and $92 \%(77 / 85)$ of the MS group $(p=1.00)$ (Table 3$)$.

Procedure-related adverse events within 7 days, including pancreatitis, acute cholangitis, acute cholecystitis, and death, were observed in $8 \%$ of the MS group versus $0 \%$ of the iPS group (Table 3). One patient in the MS group died of rapid cancerous debility, but it was unclear if it was related to the procedure. No cases of hemorrhage or intestinal perforation occurred in either group.

\section{Time to recurrence of biliary obstruction}

During the mean follow-up period of $203 \pm 224$ days for the iPS group and $182 \pm 195$ days for the MS group, RBO was observed in $21 \%(4 / 19)$ and $43 \%(33 / 77)$ of patients, respectively (Table 3 ). The median time to RBO calculated using the Kaplan-Meier method was 561 (95\% CI, 0-1,186) and 209 (95\% CI, 127-291) days, respectively, showing a statistically significant difference ( $p=0.008$ ) (Fig. 2). Re-intervention for RBO was performed for $4(21 \%)$ and $28(36 \%)$ patients in the iPS and MS groups, respectively, with the use of endoscopic ultrasonography-guided hepaticogastrostomy in 5 MS-group patients and percutaneous biliary drainage in $1 \mathrm{MS}$-group patient. Two or fewer re-interventions were performed for all iPS patients, whereas three or more re-interventions were required for 10 patients (11\%) in the MS group, although the overall survival was similar. Median time to RBO tended to
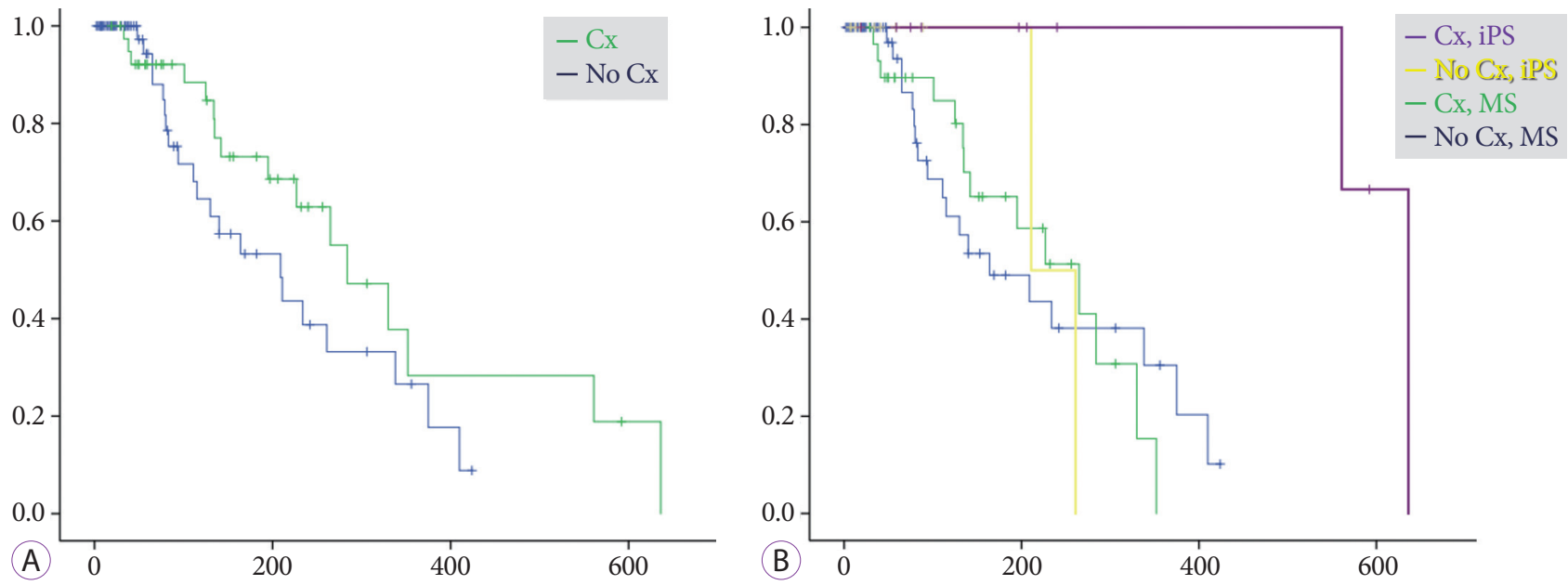

Fig. 3. Kaplan-Meier curves of cumulative rates to recurrent biliary obstruction (RBO) for patients who did versus did not undergo chemotherapy (Cx). (A) Median times to RBO (TRBO) were 284 (95\% confidence interval [Cl], 195-373) days for patients who underwent $\mathrm{Cx}$ after the procedure and 209 (95\% Cl, 112-306) days for patients who did not $(p=0.13)$. (B) For the inside plastic stent (iPS) group, median TRBO times were 636 days $(95 \% \mathrm{Cl}$, not available) for patients who underwent $\mathrm{Cx}$ and 211 days $(95 \% \mathrm{Cl}$, not available) for those who did not; for the metal stent (MS) group, they were $265(95 \% \mathrm{Cl}, 162-368)$ days and 164 (95\% Cl, 54-274) days $(p=0.028)$. 
be long for patients who underwent chemotherapy after the procedure (Fig. 3). Survival periods did not differ between the iPS and MS groups, although they were significantly different between patients who underwent chemotherapy and those who did not (Fig. 4).

\section{DISCUSSION}

A meta-analysis of three randomized controlled trials (RCTs) evaluating outcomes of using PS vs. MS for malignant perihilar obstructions concluded that MS placement was favorable with a significant difference in patency period $(p=0.003),{ }^{4}$ although two of the analyzed studies did not indicate a statistically significant difference in patency. ${ }^{1,2}$ However, the meta-analysis included cases treated using a single stent, and our retrospective cohort study included the use of two or more stents and had similar outcomes (median stent patency period: 115 days for the PS group vs. 250 days for the MS group, $p=0.0061){ }^{12}$ According to currently available data, MSs are superior to PSs for treating malignant perihilar obstructions.

MS placement to treat malignant perihilar obstruction has been generally performed using uncovered MSs to avoid side branch occlusion. However, since uncovered stents are unremovable, stent exchange is not an option for re-intervention when the stent fails or localized cholangitis occurs in separate cavities. As the number of stents that can be placed in a small, intricate area is highly limited, treatment by stent addition often becomes difficult for recurring cholangitis, decreasing the patient's QOL and possibly, survival period. ${ }^{13}$ The removability of PSs is a strong advantage and enables the exchange for new stents without bile sludge and biofilm related to occlusion recurrence. Moreover, PSs are never occluded by tumor ingrowth. Despite their short patency, PSs have several advantages in cases of perihilar obstructions, and given its replaceability, PSs remain a reasonable option.

All three previous randomized studies on the use of PS vs. MS to treat malignant perihilar obstructions compared transpapillary PS and suprapapillary MS placement. ${ }^{1-3}$ PSs across the papilla cause continuous reflux of duodenal fluids related to bacterial exposure, outlet obstruction, and sludge formation. Although no randomized studies have investigated malignant perihilar obstructions in humans, suprapapillary placement resulted in fewer occluded stents in a previous experiment on dogs. ${ }^{14}$ Moreover, in a retrospective study on humans by Inatomi et al., suprapapillary PS placement had more favorable outcomes than transpapillary PS placement (median patency period: 142 for iPSs vs. 32 for transpapillary PSs, $p=0.04$ on log rank test). ${ }^{10}$

This is the first study evaluating outcomes of suprapapillary PS versus suprapapillary MS placement. However, three retrospective studies of iPS without comparison to MS reported median patency periods of 136 days, ${ }^{9} 142$ days, ${ }^{10}$ and 190 days. ${ }^{8}$ In our study, iPSs had significantly longer median patency periods with no unfavorable outcomes in terms of clinical efficacy, short- and long-term adverse events, or survival period. However, these data, which apparently support the overwhelming superiority of iPS placement, must be confirmed in future prospective randomized studies with larger populations.

It is unknown why the patency was much longer here than in previous studies. There are several possible biases, including selection bias, derived from the retrospective nature of this study. The doctors might have consciously or unconsciously
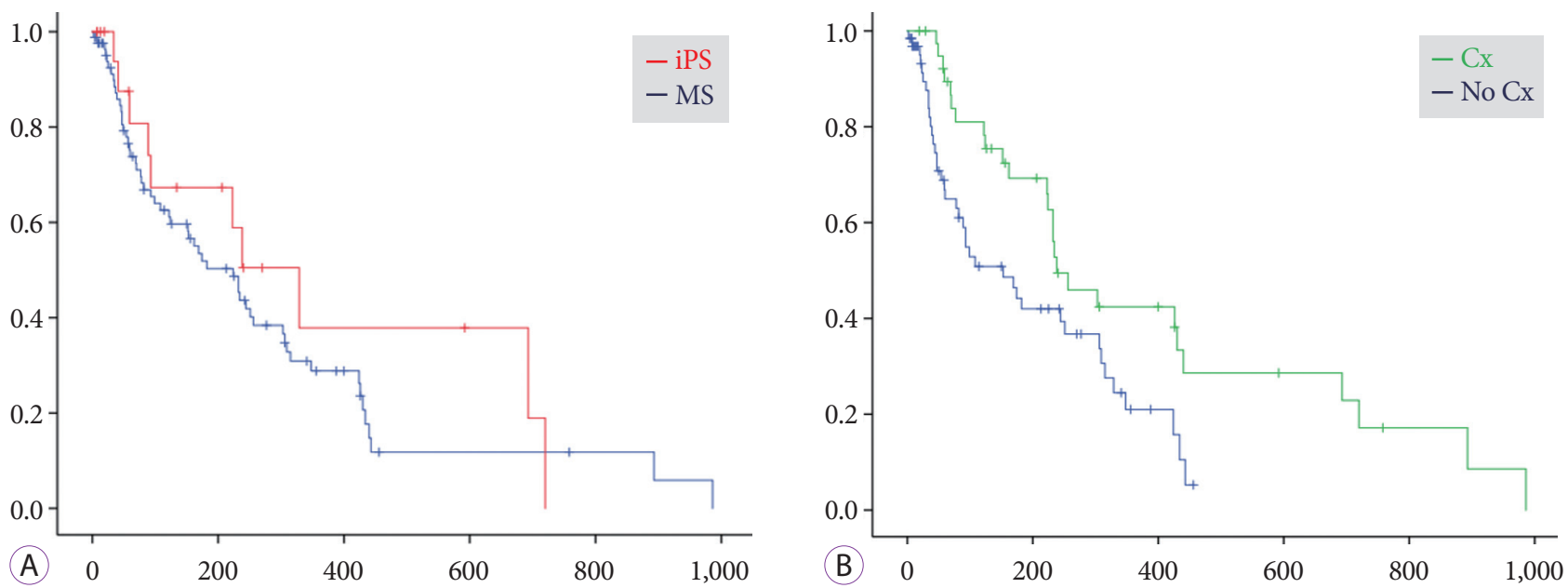

Fig. 4. Kaplan-Meier curves indicating cumulative survival rates. (A) Median survival periods were $329(95 \%$ confidence interval [Cl], 181-477) days for the inside plastic stent (iPS) group and $224(95 \% \mathrm{Cl}, 149-298)$ days for the metal stent (MS) group ( $p=0.37)$. (B) Median survival periods were $238(95 \% \mathrm{Cl}, 148-328)$ days for patients who underwent chemotherapy $(\mathrm{Cx})$ and $153(95 \% \mathrm{Cl}, 74-232)$ days for those who did not $(p=0.011)$. 
selected the stent type appropriate for the stricture. However, no statistically significant differences were found in the analyses of the baseline characteristics, including Bismuth level. Moreover, selection seems difficult because no factors related to a long iPS patency have been identified in previous reports, commentaries, and publications, although such factors must be clarified.

Another possible reason is the difference in conditions among the studies. In a well-designed retrospective study by Inatomi et al. patients with lower Bismuth levels (types I and II) were included and appeared to be associated with stent patencies of less than 60 days. ${ }^{10}$ As suggested in an RCT by Pedersen et al. in which suprapapillary and transpapillary PSs (using a hybrid endoscopic-percutaneous technique) were compared for treating obstructions of the extrahepatic (not perihilar) bile duct, ${ }^{15}$ iPSs might not be favorable for non-perihilar strictures. Strictures in the non-hilar-involving bile duct can be related to shorter functional periods of iPSs due to stent location instability, which can cause wedging of the distal (duodenal side) end to the lower narrow bile duct. In Kaneko et al., completely straight stents without angles were used, possibly resulting in a relatively short time to RBO (median, 190 days) without patients with a stent patency exceeding 400 days. $^{8}$ Although the median time to RBO in Ishiwatari's study was shorter (134 days) with curved stents and no patients with lower Bismuth levels, there were 3 patients in whom the stent functioned for longer than 1 year $(>600$ days in one patient and $>800$ days in another patient). ${ }^{9}$ Since studies on iPSs for use with malignant perihilar obstructions, including our study, included small populations, the statistical reliability of estimated median values is low. Further prospective evaluations of large populations are required.

In conclusion, the stent functioning periods of PSs placed inside the bile duct were significantly longer than those of MSs in the retrospective setting with a relatively small population size. Although the value of iPSs should be confirmed in future studies and methodologies, including appropriate shapes, should be established, the importance of removable stents will increase in this era in which curative chemotherapy can occur and conversion surgery becomes possible.

\section{Conflicts of Interest}

The authors have no financial conflicts of interest.

\section{Author Contributions}

\section{Conceptualization: Yoshihide Kanno}

Formal analysis: Shinsuke Koshita, Takahisa Ogawa, Hiroaki Kusunose, Kaori Masu, Toshitaka Sakai, Fumisato Kozakai, Yutaka Noda

Investigation: SK, TO, HK, KM, TS, Keisuke Yonamine, Kazuaki Miyamoto, Toji Murabayashi, FK, Jun Horaguchi, YN

Supervision: Kei Ito

Validation: KI
Writing-original draft: YK

Writing-review\&editing: KI

ORCID

Shinsuke Koshita: https://orcid.org/0000-0001-6880-5532 Takahisa Ogawa: https://orcid.org/0000-0002-8934-6555 Hiroaki Kusunose: https://orcid.org/0000-0002-8563-9342 Kaori Masu: https://orcid.org/0000-0003-2366-8700 Toshitaka Sakai: https://orcid.org/0000-0002-7450-8478 Keisuke Yonamine: https://orcid.org/0000-0003-1121-7320 Kazuaki Miyamoto: https://orcid.org/0000-0001-8313-1396 Toji Murabayashi: https://orcid.org/0000-0001-8604-8125 Fumisato Kozakai: https://orcid.org/0000-0002-5890-3531 Jun Horaguchi: https://orcid.org/0000-0003-3987-0639 Yutaka Noda: https://orcid.org/0000-0001-6568-6590 Kei Ito: https://orcid.org/0000-0002-0023-7919

\section{REFERENCES}

1. Wagner HJ, Knyrim K, Vakil N, Klose KJ. Plastic endoprostheses versus metal stents in the palliative treatment of malignant hilar biliary obstruction. A prospective and randomized trial. Endoscopy 1993;25:213218.

2. Sangchan A, Kongkasame W, Pugkhem A, Jenwitheesuk K, Mairiang P. Efficacy of metal and plastic stents in unresectable complex hilar cholangiocarcinoma: a randomized controlled trial. Gastrointest Endosc 2012;76:93-99.

3. Mukai T, Yasuda I, Nakashima M, et al. Metallic stents are more efficacious than plastic stents in unresectable malignant hilar biliary strictures: a randomized controlled trial. J Hepatobiliary Pancreat Sci 2013;20:214-222.

4. Zorrón Pu L, de Moura EG, Bernardo WM, et al. Endoscopic stenting for inoperable malignant biliary obstruction: a systematic review and meta-analysis. World J Gastroenterol 2015;21:13374-13385.

5. Kurita A, Kodama Y, Minami R, et al. Endoscopic stent placement above the intact sphincter of Oddi for biliary strictures after living donor liver transplantation. J Gastroenterol 2013;48:1097-1104.

6. Tsujino T, Isayama H, Kogure H, Sato T, Nakai Y, Koike K. Endoscopic management of biliary strictures after living donor liver transplantation. Clin J Gastroenterol 2017;10:297-311.

7. Kobayashi N, Watanabe S, Hosono K, et al. Endoscopic inside stent placement is suitable as a bridging treatment for preoperative biliary tract cancer. BMC Gastroenterol 2015;15:8.

8. Kaneko T, Sugimori K, Shimizu Y, et al. Efficacy of plastic stent placement inside bile ducts for the treatment of unresectable malignant hilar obstruction (with videos). J Hepatobiliary Pancreat Sci 2014;21:349-355.

9. Ishiwatari H, Hayashi T, Ono M, Sato T, Kato J. Newly designed plastic stent for endoscopic placement above the sphincter of Oddi in patients with malignant hilar biliary obstruction. Dig Endosc 2013;25 Suppl 2:94-99.

10. Inatomi O, Bamba S, Shioya $\mathrm{M}$, et al. Threaded biliary inside stents are a safe and effective therapeutic option in cases of malignant hilar obstruction. BMC Gastroenterol 2013;13:31.

11. Kanno Y, Ito K, Koshita S, et al. Soft surface irregularity of malignant perihilar biliary strictures in cholangiography as a risk factor for early dysfunction of multiple metal stents. Dig Liver Dis 2017;49:1014-1021.

12. Kanno Y, Ito K, Fujita N, et al. Single-session endoscopic bilateral y-configured placement of metal stents for hilar malignant biliary obstruction. Dig Endosc 2011;23:91-96.

13. Kanno Y, Ito K, Koshita S, et al. EUS-guided biliary drainage for malignant perihilar biliary strictures after further transpapillary intervention has been judged to be impossible or ineffective. Intern Med 


\section{$C_{\text {cumban Enossoory }}$}

2017;56:3145-3151.

14. Geoghegan JG, Branch MS, Costerton JW, Pappas TN, Cotton PB. Placement of biliary stents above the sphincter of Oddi prolongs stent patency in dogs. Gut 1991;32:A1232.
15. Pedersen FM, Lassen AT, Schaffalitzky de Muckadell OB. Randomized trial of stent placed above and across the sphincter of Oddi in malignant bile duct obstruction. Gastrointest Endosc 1998;48:574-579. 